\title{
ORIGINAL
}

\section{CARACTERIZACIÓN DEL AMBIENTE ATMOSFÉRICO EN LAS PALMAS DE GRAN CANARIA Y SANTA CRUZ DE TENERIFE. 2000 A 2004}

\author{
Elena López Villarrubia (1), M Dolores García Pérez (1), Nieves Peral Pérez (1,2), Ferrán \\ Ballester Díez (2,3), Carmen Iñiguez Fernández (2,3), M Luisa Pita Toledo (1) \\ (1) Dirección General de Salud Pública. Gobierno de Canarias. \\ (2) Escuela Valenciana de Estudios en Salud (EVES). \\ (3) CIBER en epidemiología y salud pública (CIBERESP).
}

\section{RESUMEN}

Fundamento: El carácter insular de las ciudades de Las Palmas de Gran Canaria y Santa Cruz de Tenerife, su meteorología y la proximidad del continente africano que favorece la llegada de material particulado de origen natural sobre las islas, determinan unas especificidades en su calidad del aire. El objetivo de este artículo es la caracterización de la contaminación atmosférica durante los años 2000 a 2004 como indicador de exposición de los habitantes de estas dos ciudades.

Métodos: Se elaboraron los siguientes indicadores de contaminación: promedios de 24 horas de $\mathrm{PM}_{10}, \mathrm{PM}_{2,5}, \mathrm{NO}_{2}, \mathrm{SO}_{2}$ y $\mathrm{O}_{3}$ máximo de las 17 medias móviles octohorarias diarias de $\mathrm{O}_{3}$ y CO; máximo horario diario de $\mathrm{SO}_{2}, \mathrm{NO}_{2}, \mathrm{O}_{3}, \mathrm{CO}, \mathrm{PM}_{10}$ y $\mathrm{PM}_{25}$. También se calcularon los niveles de la moda gruesa de partículas, como la diferencia entre los valores de $\mathrm{PM}_{10}$ y $\mathrm{PM}_{2,5}$ $\left(\mathrm{PM}_{10-25}\right)$. Se identificaron episodios de intrusión africana.

Resultados: En Sta Cruz de TF los promedios de $\mathrm{SO}_{2}$ $\left(14 \mu \mathrm{g} / \mathrm{m}^{3} \mathrm{~N}\right)$ y de $\mathrm{O}_{3}\left(44,4 \mu \mathrm{g} / \mathrm{m}^{3} \mathrm{~N}\right)$ fueron superiores respecto a los de Las Palmas de GC ( 8 y $28,3 \mu \mathrm{g} / \mathrm{m}^{3} \mathrm{~N}$ ). Los promedios de $\mathrm{NO}_{2}$ en Las Palmas de GC: $45,8 \mu \mathrm{g} / \mathrm{m}^{3} \mathrm{~N}$ fueron superiores a los de Sta. Cruz de TF: $30,3 \mu \mathrm{g} / \mathrm{m}^{3} \mathrm{~N}$. Debido a las intrusiones africanas, algunos días superaron los $600 \mu \mathrm{g} / \mathrm{m}^{3} \mathrm{de}$ $\mathrm{PM}_{10}$ y los 200 de $\mathrm{PM}_{25}$ en ambas ciudades.

Conclusiones: Se identifica un patrón de calidad del aire con episodios de polvo mineral africano que en superficie afecta a todas las fracciones granulométricas, una estacionalidad diferente a las ciudades europeas respecto al ozono, así como un patrón de contaminación urbano-industrial en Sta. Cruz de Tf y netamente urbano en Las Palmas de GC. Se hace necesario tener en cuenta estos resultados para analizar su posible impacto sobre la salud de los ciudadanos de las Islas Canarias y establecer sistemas adecuados de vigilancia.

Palabras clave: Contaminación atmosférica. Dióxido de azufre. Dióxido de nitrógeno. Ozono. Monóxido de carbono.

Correspondencia:

Elena López Villarrubia

Dirección General de Salud Pública

c/ Alfonso XIII, 4.

35003 Las Palmas de Gran Canaria

Tf: 928452248

Fax: 928452210

elopvil@gobiernodecanarias.org

\section{ABSTRACT}

\section{Characterization of the atmospheric environment in Las Palmas de Gran Canaria and Santa Cruz de Tenerife, Spain. 2000-2004}

Background: The island factor in the cities of Las Palmas de Gran Canaria and Santa Cruz de Tenerife, their meteorology and the proximity to the African Continent that originates the natural particulate matter transport over the islands, cause some specific features in their air quality. The aim of this paper is to characterize the air pollution from 2000 to 2004 as exposure indicator of both cities' inhabitants.

Methods: 24 hour daily average variables of $\mathrm{PM}_{10}, \mathrm{PM}_{2,5}$, $\mathrm{NO}_{2}, \mathrm{SO}_{2}$ and $\mathrm{O}_{3}, 8$ hours daily maxima moving averages of $\mathrm{O}_{3}$ y CO and 1 hour maxima of $\mathrm{SO}_{2}, \mathrm{NO}_{2}, \mathrm{O}_{3}, \mathrm{CO}, \mathrm{PM}_{10} \mathrm{y}$ $\mathrm{PM}_{25}$ were calculated. Daily levels of coarse particles were obtained subtracting $\mathrm{PM}_{2,5}$ from $\mathrm{PM}_{10}$. African dust events were identified.

Results: In Sta. Cruz de Tf daily means of $\mathrm{SO}_{2}$ $\left(14.0 \mu \mathrm{g} / \mathrm{m}^{3} \mathrm{~N}\right)$ and ozone levels $\left(44.4 \mu \mathrm{g} / \mathrm{m}^{3} \mathrm{~N}\right)$ were higher than Las Palmas de GC levels ( 8.0 y $28.3 \mu \mathrm{g} / \mathrm{m}^{3} \mathrm{~N}$ ). Daily means of $\mathrm{NO}_{2}$ in Las Palmas de GC: $45.8 \mu \mathrm{g} / \mathrm{m}^{3} \mathrm{~N}$ where higher than Sta. Cruz de Tf levels: $30.3 \mu \mathrm{g} / \mathrm{m}^{3} \mathrm{~N}$. Due to African dust outbreaks, some days in both cities exceeded 600 $\mu \mathrm{g} / \mathrm{m}^{3}$ of $\mathrm{PM}_{10}$ and 200 of $\mathrm{PM}_{2.5} 24-\mathrm{h}$ average.

Conclusions: The air quality patterns were characterized by very high levels of African dust outbreaks that affect all PM size fractions. Different $\mathrm{O}_{3}$ seasonality exists respect European cities in addition to an urban-industrial ambient air in Sta. Cruz de TF and clearly urban in Las Palmas de GC. These results have to be considered in order to lay the foundations to suitable surveillance systems, analyse the potential impact on the Canary Islands citizens' health and to get conclusions.

Key words: Air pollution. $\mathrm{PM}_{10}, \mathrm{PM}_{2,5}$, Sulphur dioxide. Nitrogen dioxide. Ozone. carbon monoxide.

Proyecto financiado por la Fundación Canaria de Investigación y Salud (FUNCIS) PI 57/05 


\section{INTRODUCCIÓN}

La situación de las Islas Canarias en el eje de la franja subtropical y al oeste del continente africano, les confiere unos rasgos climáticos específicos. El anticiclón de las Azores genera en las islas un régimen de vientos alisios, con una estructura vertical, que en su componente inferior sopla de Noreste y Norte-Noroeste. Al discurrir a nivel del mar estos vientos son frescos y húmedos y al soplar de forma casi constante de mayo a octubre, favorecen la dispersión de los contaminantes primarios emitidos en los entornos urbanos ubicados a barlovento. A su vez, la corriente fría del Golfo de México enfría las capas de aire en contacto con el mar, por lo que el clima subtropical que correspondería por su latitud geográfica queda moderado y suavizado. Por otra parte, la implantación del anticiclón norteafricano en superficie produce un continuado aporte de polvo desde el norte de África hacia América, pasando sobre Canarias. En verano este transporte tiene lugar a alturas considerables, afectando a ciudades costeras por deposición gravitacional desde niveles altos, mientras que en invierno, con el incremento de la altura de la capa de inversión térmica, el transporte tiene lugar en la capa límite marítima ${ }^{1}$.

Todas las circunstancias anteriores dan como resultado unas características particulares en la calidad del aire que respiran las personas.

El objetivo de este artículo es la caracterización de la contaminación atmosférica durante el periodo de estudio comprendido entre los años 2000 a 2004 como indicador de exposición de los habitantes de estas dos ciudades.

\section{MATERIAL Y MÉTODO}

Las Palmas de Gran Canarias se ubica en el cuadrante Noreste de la isla de Gran
Canaria y Sta. Cruz de Tenerife en el extremo oriental de la isla de Tenerife, ambas junto al mar (figura 1), de manera que se encuentran bajo la influencia directa de los vientos alisios, húmedos y constantes. Las dos ciudades se caracterizan por presentar temperaturas suaves (temperaturas medias de 19 a $23{ }^{\circ} \mathrm{C}$ ) y escasa oscilación térmica, con precipitaciones muy escasas e irregulares. No obstante, las características de sus respectivos entornos marcan ciertas diferencias ambientales entre ellas.

La ciudad de Las Palmas de GC, a 150 $\mathrm{Km}$ de la costa africana y con una población estimada a mitad de periodo de estudio cercana a los 374.000 habitantes, se encuentra en gran parte asentada sobre un istmo, lo que permite una cómoda circulación de los alisios desde el noreste, facilitando las condiciones dispersivas de forma habitual. Alberga cerca del $50 \%$ de la población de toda la isla y tiene una densidad de $3.751,4 \mathrm{hab} . / \mathrm{Km}^{2}$. La fuente principal de contaminantes antropogénicos es el tráfico rodado.

En Sta. Cruz de TF, con una población cercana a los 219.000 habitantes, las condiciones dispersivas se pueden ver disminuidas en presencia de vientos del este-sureste debido a que el Macizo de Anaga la limita al Noroeste. Por otro lado, además de la influencia derivada del tráfico, esta ciudad también soporta la contaminación originada por la industria de fraccionamiento y separación de productos derivados del petróleo.

Datos. La información sobre contaminación atmosférica se ha obtenido de la Red de Vigilancia y Control de la Calidad del Aire. Para este estudio se han seleccionado dos estaciones en Sta. Cruz de TF: Tomé Cano y Los Gladiolos, y una en Las Palmas de Gran Canaria: Mercado Central. Estas estaciones presentaron datos válidos en más del $94 \%$ de los días para 

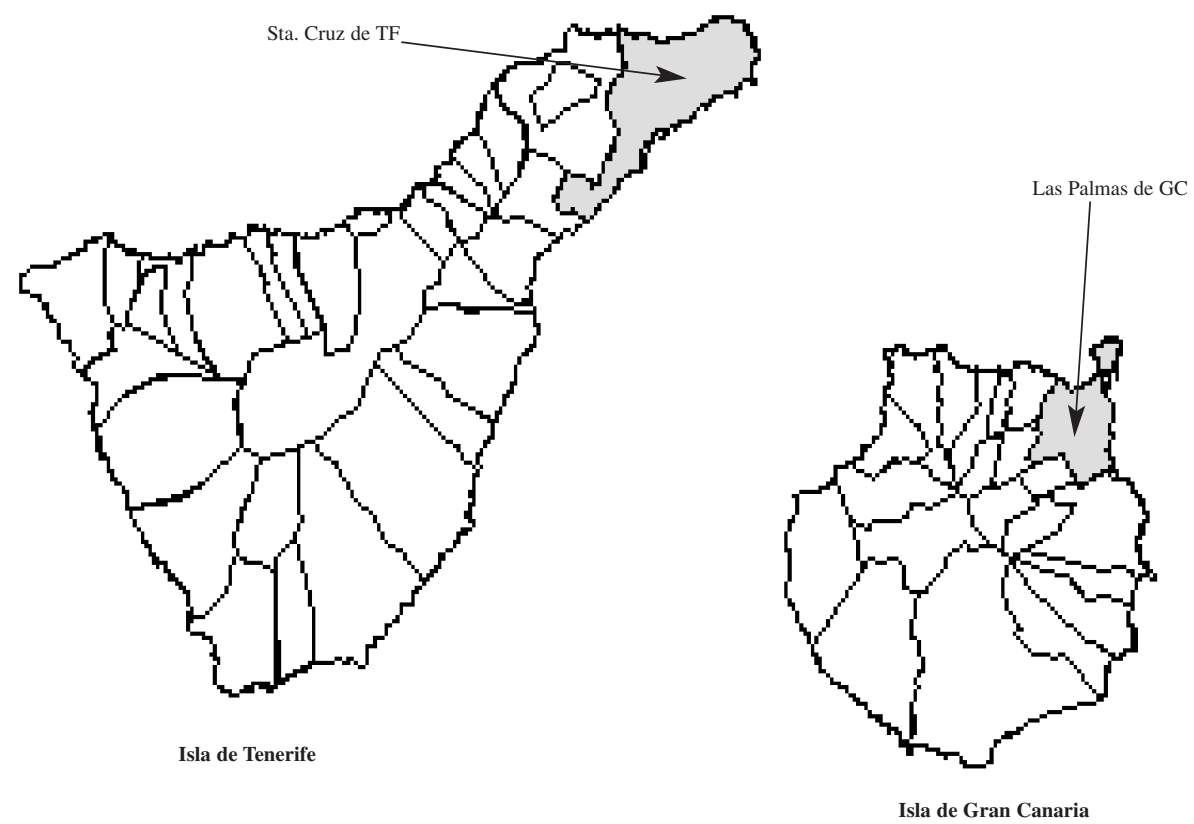

los contaminantes analizados. Esta red registra en tiempo real los valores horarios de dióxido de azufre (fluorescencia ultravioleta), dióxido de nitrógeno (quimiluminiscencia), partículas de diámetro inferior a 10 micras $\left(\mathrm{PM}_{10}\right)$ (determinación gravimétrica y radiación $\beta$ ), partículas de diámetro inferior a 2,5 micras $\left(\mathrm{PM}_{2,5}\right)$ (muestreo por aspiración en determinación gravimétrica), ozono (absorción ultravioleta) y monóxido de carbono (absorción infrarroja).

Con los valores horarios representativos de cada ciudad se construyeron las siguientes variables: promedios de 24 horas de $\mathrm{PM}_{10}, \mathrm{PM}_{2,5}$, dióxido de nitrógeno $\left(\mathrm{NO}_{2}\right)$, dióxido de azufre $\left(\mathrm{SO}_{2}\right)$ y ozono $\left(\mathrm{O}_{3}\right)$ máximo de las 17 medias móviles octohorarias diarias de $\mathrm{O}_{3}$ y monóxido de carbono (CO); máximo horario diario de $\mathrm{SO}_{2}, \mathrm{NO}_{2}, \mathrm{O}_{3}$ ,CO, $\mathrm{PM}_{10}$ y $\mathrm{PM}_{2,5}$. También se calcularon los niveles de la moda gruesa de partículas, como la diferencia entre los valores de $\mathrm{PM}_{10}$ y $\mathrm{PM}_{2,5}\left(\mathrm{PM}_{10-2,5}\right)$.

Identificación de los episodios de intrusión africana. En cada ciudad se identificaron los días que sobrepasaban los $50 \mu \mathrm{g} / \mathrm{m}^{3}$ de promedio diario de $\mathrm{PM}_{10}$ y coincidían con los días clasificados como episódicos en todas las islas (según protocolo del Instituto Nacional de Meteorología, el Ministerio de Medio Ambiente y el $\operatorname{CSIC}^{2,3}$ ) o dos días antes y hasta cuatro después. El motivo es que suele haber un cierto inicio retardado de los distintos episodios de una ciudad respecto a otra según el origen de la intrusión. A ello se añade que siempre puede quedar una cierta residencia de partículas a las que sigue expuesta la población en función de las condiciones meteorológicas subsiguientes al evento (si las masas de aire no 
son sucedidas por episodios de advección atlántica intensa que remuevan las masas de aire).

\section{RESULTADOS}

En la tabla 1 se resumen las medidas descriptivas de los indicadores de contaminación y las variables meteorológicas en las dos ciudades a estudio.

El número de días identificados como episodio de intrusión de polvo africano fue de 210 en Las Palmas de GC y 283 en Sta. Cruz de TF, lo que representa el 11,5 y el $15,5 \%$ de los días, respectivamente. Los niveles de $\mathrm{PM}_{10}$ y $\mathrm{PM}_{2,5}$ son similares en las dos ciudades: 37,8 y $16,4 \mu \mathrm{g} / \mathrm{m}^{3}$ en Las Palmas de GC y 42,3 y $15,2 \mu \mathrm{g} / \mathrm{m}^{3}$ en Sta
Cruz de (tabla 1). Estos promedios diarios disminuyen claramente si se excluyen de las series aquellos días clasificados como episodio, descendiendo a niveles de alrededor de $30 \mu \mathrm{g} / \mathrm{m}^{3}$ de $\mathrm{PM}_{10}$ y $12-13 \mu \mathrm{g} / \mathrm{m}^{3}$ de $\mathrm{PM}_{2,5}$ en las dos ciudades, es decir una diferencia de unos $10 \mu \mathrm{g} / \mathrm{m}^{3}$ de PM10 y $4 \mu \mathrm{g} / \mathrm{m}^{3}$ de PM2.5 (tabla 2). La figura 2 muestra los niveles mensuales de $\mathrm{PM}_{10}$ y $\mathrm{PM}_{2,5}$ en cada una de las ciudades, observándose que ambos son más altos durante los meses de invierno. En general los valores de $\mathrm{PM}_{10}$, son ligeramente superiores en Sta. Cruz de Tenerife y los de $\mathrm{PM}_{2,5}$ en Las Palmas de GC (tabla 1).

La media diaria de $\mathrm{SO}_{2}$ en Sta. Cruz de $\mathrm{TF}\left(14,1 \mu \mathrm{g} / \mathrm{m}^{3} \mathrm{~N}\right)$ es superior a la de Las Palmas de GC $\left(8,1 \mu \mathrm{g} / \mathrm{m}^{3} \mathrm{~N}\right.$, y sus valores máximos $\left(145,9 \mu \mathrm{g} / \mathrm{m}^{3} \mathrm{~N}\right)$ se aproximan a

Tabla 1

Estadísticos descriptivos de los indicadores diarios de exposición a la contaminación y variables meteorológicas: 2000 a 2004 (expresado en $\mu \mathrm{g} / \mathrm{m}^{3}$, excepto CO expresado en $\mathrm{mg} / \mathrm{m}^{3}$ )

\begin{tabular}{|l|c|c|c|c|c|c|c|c|c|c|}
\hline \multirow{2}{*}{$\begin{array}{c}\text { Indicador de } \\
\text { contaminación }\end{array}$} & \multicolumn{4}{|c|}{ Las Palmas de Gran Canaria } & \multicolumn{5}{c|}{ Santa Cruz de Tenerife } \\
\cline { 2 - 12 } & \% Válidos & media & dt & mínimo & máximo & \% Válidos & media & dt & mínimo & máximo \\
\hline $\mathrm{PM}_{10}-24 \mathrm{~h}$ & 95,5 & 37,8 & 40,6 & 8,7 & 612,4 & 99,3 & 42,3 & 41,7 & 13,0 & 622,1 \\
\hline $\mathrm{PM}_{10}-1 \mathrm{~h}$ & 95,5 & 70,4 & 77,8 & 14,1 & 998,7 & 99,3 & 81,72 & 88,6 & 18,5 & 1002,2 \\
\hline $\mathrm{PM}_{25}-24 \mathrm{~h}$ & 98,2 & 16,4 & 15,2 & 2,42 & 10,5 & 95,3 & 15,1 & 14,3 & 3,5 & 227,6 \\
\hline $\mathrm{PM}_{25}-1 \mathrm{~h}$ & 98,2 & 28,9 & 36,5 & 2,4 & 570,7 & 95,3 & 29,1 & 34,8 & 6,1 & 503 \\
\hline $\mathrm{PM}_{10-25}-24 \mathrm{~h}$ & 94,0 & 20,2 & 25,5 & 2,1 & 401,8 & 94,9 & 26,6 & 25,9 & 5,0 & 394,5 \\
\hline $\mathrm{PM}_{10-25}-1 \mathrm{~h}$ & 94,0 & 43,5 & 55,9 & 6,6 & 776,9 & 94,9 & 61,0 & 60,8 & 19,7 & 685,4 \\
\hline $\mathrm{SO}_{2}-24 \mathrm{~h}$ & 97,0 & 8,1 & 4,2 & 1,5 & 44,4 & 99,9 & 14,1 & 12,6 & 2,3 & 145,9 \\
\hline $\mathrm{SO}_{2}-1 \mathrm{~h}$ & 97,0 & 12,1 & 9,9 & 1,9 & 99,8 & 99,9 & 41,2 & 66,2 & 3,8 & 808,6 \\
\hline $\mathrm{NO}_{2}-24 \mathrm{~h}$ & 96,0 & 45,8 & 14,8 & 7,2 & 104,7 & 99,3 & 30,3 & 16,4 & 5,4 & 93,3 \\
\hline $\mathrm{NO}_{2}-1 \mathrm{~h}$ & 96,0 & 70,1 & 24,4 & 9,0 & 219,0 & 99,3 & 56,2 & 28,3 & 5,6 & 236,7 \\
\hline $\mathrm{CO}_{-24 h}$ & 95,0 & 0,6 & 0,35 & 0,1 & 4,54 & 95,7 & 0,8 & 0,5 & 0,10 & 3,3 \\
\hline $\mathrm{CO}-8 \mathrm{~h}$ & 95,0 & 0,9 & 0,6 & 0,1 & 6,8 & 95,7 & 1,1 & 0,7 & 0,1 & 4,5 \\
\hline $\mathrm{CO}-1 \mathrm{~h}$ & 95,0 & 1,3 & 0,9 & 0,2 & 11,4 & 95,7 & 1,9 & 1,3 & 0,1 & 12,8 \\
\hline $\mathrm{O}_{3}-24 \mathrm{~h}$ & 94,0 & 28,3 & 13,1 & 3,8 & 86,13 & 99,7 & 44,34 & 15,4 & 9,1 & 96,8 \\
\hline $\mathrm{O}_{3}-8 \mathrm{~h}$ & 94,0 & 37,9 & 17,4 & 4,2 & 114,8 & 99,7 & 53,0 & 15,1 & 15,0 & 101,4 \\
\hline $\mathrm{O}_{3}-1 \mathrm{~h}$ & 94,0 & 49,8 & 22,3 & 5,9 & 168,3 & 99,7 & 61,6 & 17,3 & 16,4 & 383,8 \\
\hline Temperatura & 100,0 & 21,1 & 2,6 & 14,4 & 32,5 & 100,0 & 21,3 & 3 & 13,9 & 33,6 \\
\hline $\mathrm{Humedad}$ & 100,0 & 65,5 & 9,4 & 22,3 & 95,0 & 100,0 & 62,2 & 7,6 & 31,3 & 85,8 \\
\hline
\end{tabular}

dt: desviación típica

$\mathrm{PM}_{10}-24 \mathrm{~h}, \mathrm{PM}_{25}-24 \mathrm{~h}, \mathrm{PM}_{10-25}-24 \mathrm{~h}, \mathrm{SO}_{2}-24 \mathrm{~h}, \mathrm{CO}-24 \mathrm{~h}, \mathrm{O}_{3}-24 \mathrm{~h}$ : promedio diario de $P M_{10}, \mathrm{PM}_{2,5}, \mathrm{PM}_{10-25}, S_{2}, C O$ y $O_{3}$. $\mathrm{PM}_{10}^{10}-1 \mathrm{~h}, \mathrm{PM}_{25}-1 \mathrm{~h}, \mathrm{PM}_{10-25}-1 \mathrm{~h}, \mathrm{SO}_{2}-1 \mathrm{~h}, \mathrm{NO}_{2}-1 \mathrm{~h}, \mathrm{CO}-1 \mathrm{~h}, \mathrm{O}_{3}-1 \mathrm{~h}$ : máximo valor horario diario.

$\mathrm{CO}-8 \mathrm{~h}, \mathrm{O}_{3}-8 \mathrm{~h}$ : máximo valor de las medias octohorarias diarias. 
Tabla 2

Promedios de los valores contaminantes durante los días sin influencia de polvo africano y aquellos con episodios de polvo africano $\mu \mathrm{g} / \mathrm{m}^{3}\left(\mathrm{CO} \mathrm{mg} / \mathrm{m}^{3}\right) .2000$ a 2004

\begin{tabular}{|l|c|c|c|c|}
\hline \multirow{2}{*}{} & \multicolumn{2}{|c|}{ Las Palmas de Gran Canaria } & \multicolumn{2}{c|}{ Santa Cruz de Tenerife } \\
\cline { 2 - 5 } & No episodio & Episodio & No episodio & 102,4 \\
\hline $\mathrm{PM}_{10}-24 \mathrm{~h}$ & 28,6 & 105,7 & 59,7 & 200,9 \\
\hline $\mathrm{PM}_{10}-1 \mathrm{~h}$ & 54,0 & 192,1 & 11,5 & 35,9 \\
\hline $\mathrm{PM}_{2.5}-24 \mathrm{~h}$ & 13,40 & 41,46 & 21,6 & 71,6 \\
\hline $\mathrm{PM}_{25}-1 \mathrm{~h}$ & 22,7 & 82,2 & 20,2 & 62,6 \\
\hline $\mathrm{PM}_{10-25}-24 \mathrm{~h}$ & 14,8 & 63,5 & 47,7 & 135,6 \\
\hline $\mathrm{PM}_{10-25}-1 \mathrm{~h}$ & 33,0 & 128,7 & 12,5 & 22,3 \\
\hline $\mathrm{SO}_{2}-24 \mathrm{~h}$ & 7,9 & 9,95 & 33,9 & 81 \\
\hline $\mathrm{SO}_{2}-1 \mathrm{~h}$ & 11,6 & 15,8 & 28,0 & 42,6 \\
\hline $\mathrm{NO}_{2}-24 \mathrm{~h}$ & 45,2 & 50,7 & 52,1 & 78,2 \\
\hline $\mathrm{NO}_{2}-1 \mathrm{~h}$ & 68,7 & 81,2 & 0,8 & 1,0 \\
\hline $\mathrm{CO}-24 \mathrm{~h}$ & 0,6 & 0,6 & 1,0 & 1,5 \\
\hline $\mathrm{CO}-8 \mathrm{~h}$ & 0,9 & 0,8 & 1,7 & 2,8 \\
\hline $\mathrm{CO}-1 \mathrm{~h}$ & 1,3 & 1,3 & 46,2 & 34,1 \\
\hline $\mathrm{O}_{3}-24 \mathrm{~h}$ & 28,4 & 27,2 & 54,4 & 45,8 \\
\hline $\mathrm{O}_{3}-8 \mathrm{~h}$ & 37,9 & 38,1 & 63,6 & 56,0 \\
\hline $\mathrm{O}_{3}-1 \mathrm{~h}$ & 49,8 & 49,4 & $50^{2}$ & \\
\hline
\end{tabular}

$\mathrm{PM}_{10}-24 \mathrm{~h}, \mathrm{PM}_{25}-24 \mathrm{~h}, \mathrm{PM}_{10-25}-24 \mathrm{~h}, \mathrm{SO}_{2}-24 \mathrm{~h}, \mathrm{CO}-24 \mathrm{~h}, \mathrm{O}_{3}-24 \mathrm{~h}$ : promedio diario de $P M_{10}, P M_{2,5} \mathrm{PM}_{10-25}, S_{2}, C O$ y $O_{3}$.

$\mathrm{PM}_{10}-1 \mathrm{~h}, \mathrm{PM}_{25}-1 \mathrm{~h}, \mathrm{PM}_{10-25}-1 \mathrm{~h}, \mathrm{SO}_{2}-1 \mathrm{~h}, \mathrm{NO}_{2}-1 \mathrm{~h}, \mathrm{CO}-1 \mathrm{~h}, \mathrm{O}_{3}-1 \mathrm{~h}$ : máximo valor horario diario.

$\mathrm{CO}-8 \mathrm{~h}, \mathrm{O}_{3}-8 \mathrm{~h}$ : máximo valor de las medias octohorarias diarias.

los de ciudades más industrializadas, como Huelva u Oviedo, además el incremento medio de este contaminante los días de episodio $\left(10 \mu \mathrm{g} / \mathrm{m}^{3} \mathrm{~N}\right)$ es de mayor magnitud que en Las Palmas de GC $\left(2 \mu \mathrm{g} / \mathrm{m}^{3} \mathrm{~N}\right)$ (tabla 2).

Los distintos indicadores del $\mathrm{O}_{3}$ en Sta. Cruz de TF presentan una diferencia promedio de $15 \mu \mathrm{g} / \mathrm{m}^{3} \mathrm{~N}$ respecto a Las Palmas de GC durante el periodo de estudio (tabla 1). En ambas ciudades los valores máximos se alcanzan en la primavera (fig. 3) y durante la madrugada (figura 4).

Con la misma magnitud, pero con patrón exactamente inverso, el promedio de los niveles de $\mathrm{NO}_{2}\left(45,8 \mu \mathrm{g} / \mathrm{m}^{3} \mathrm{~N}\right)$ en Las Palmas de GC, es superior al de Sta. Cruz de TF $\left(30,3 \mu \mathrm{g} / \mathrm{m}^{3} \mathrm{~N}\right)$ debido al mayor peso del tráfico rodado. Los niveles de $\mathrm{CO}$ son similares en ambas ciudades $(0,6$ a 0,8 $\mu \mathrm{g} / \mathrm{m}^{3} \mathrm{~N}$ ), si bien en los días de episodio se incrementan en Sta Cruz de TF: 1,06 $\mu \mathrm{g} / \mathrm{m}^{3} \mathrm{~N}$ (tabla 2).

En cuanto a las tendencias de los niveles de los contaminantes respecto al año 2000, en Las Palmas de $\mathrm{GC}$ el $\mathrm{O}_{3}$ ha sufrido un incremento durante el periodo, con ligera disminución en los dos últimos años finalizando con niveles un $22 \%$ superiores respecto al primer año y un $7 \%$ las $\mathrm{PM}_{2.5}$ (Figura 5). En Sta. Cruz de TF a excepción del $\mathrm{NO}_{2}$, el resto de contaminantes se elevó el último año llegando hasta un incremento del $57 \%$ las $\mathrm{PM}_{2.5}$.

En la tabla 3 se observan los coeficientes de correlación entre las variables de contaminación, temperatura y humedad, que son débiles en general ( $r$ de Pearson $<0,45$ ), a excepción de los que relacionan las partículas y sus fracciones. Los 
Figura 2

Distribución mensual de los promedios diarios de $\mathrm{PM}_{10}$ y $\mathrm{PM}_{2,5}\left(\mu \mathrm{g} / \mathrm{m}^{3}\right)$ en las dos ciudades, 2000-2004

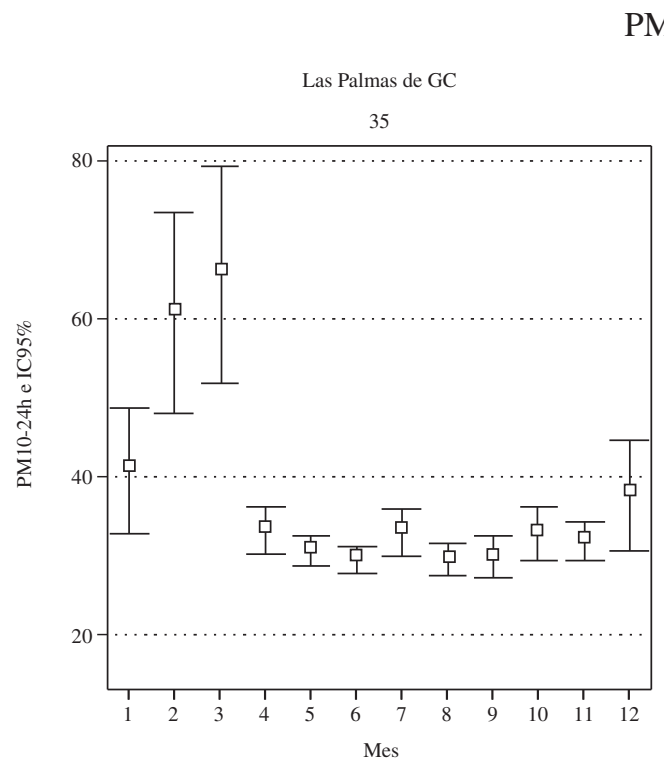

$\mathrm{PM}_{10}$
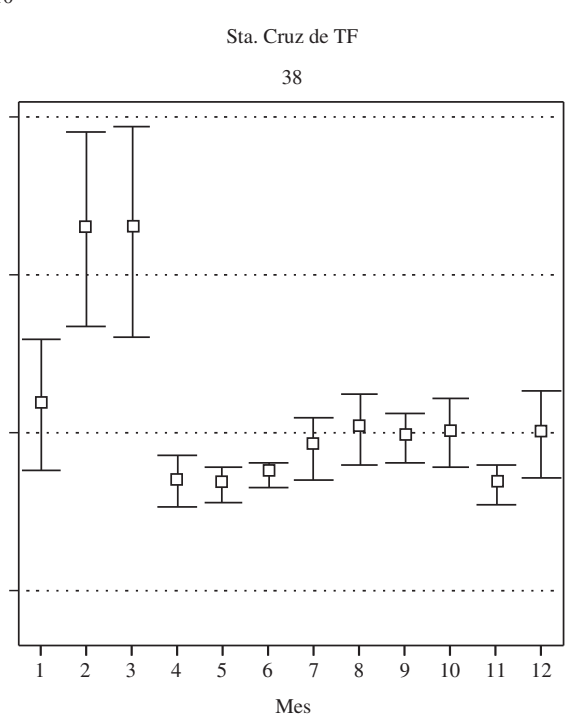

$\mathrm{PM}_{10}$

Las Palmas de GC

35

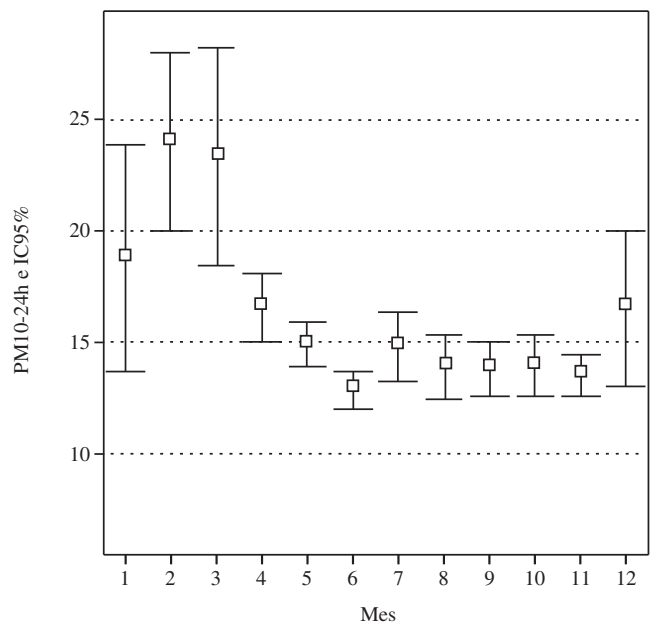

Sta. Cruz de TF

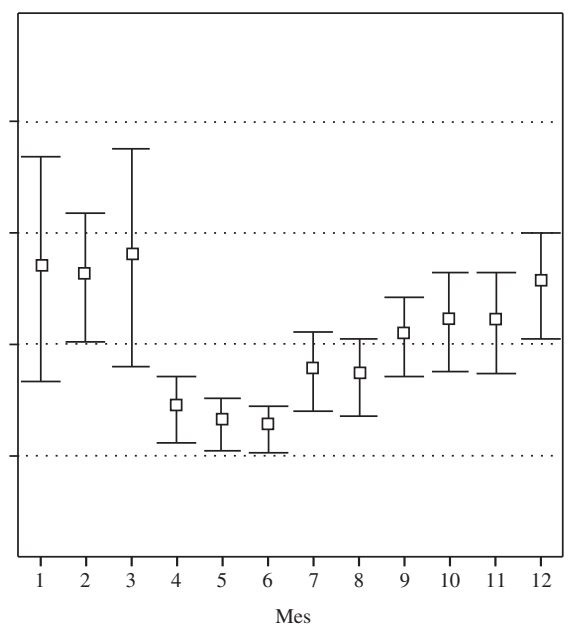




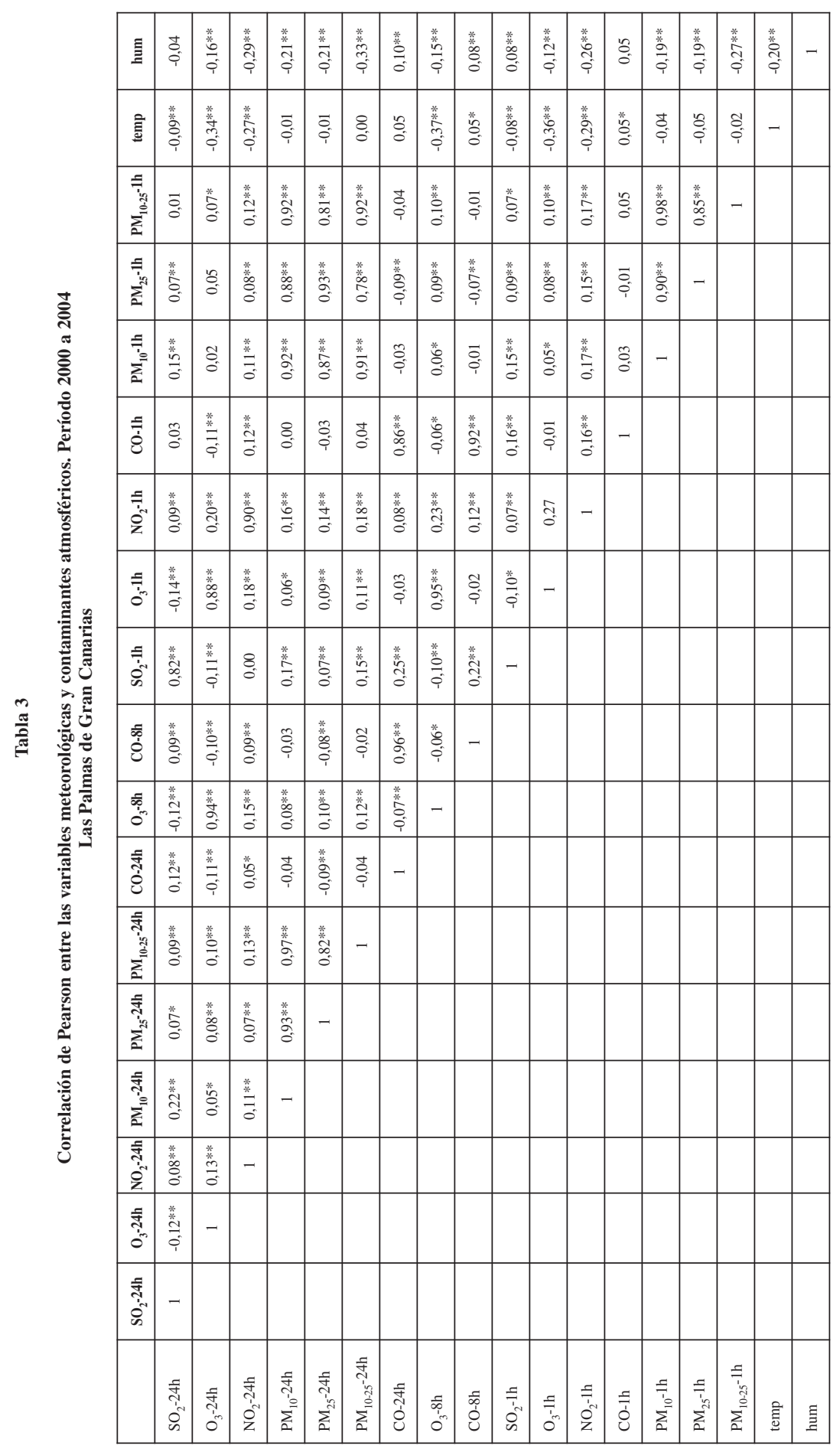




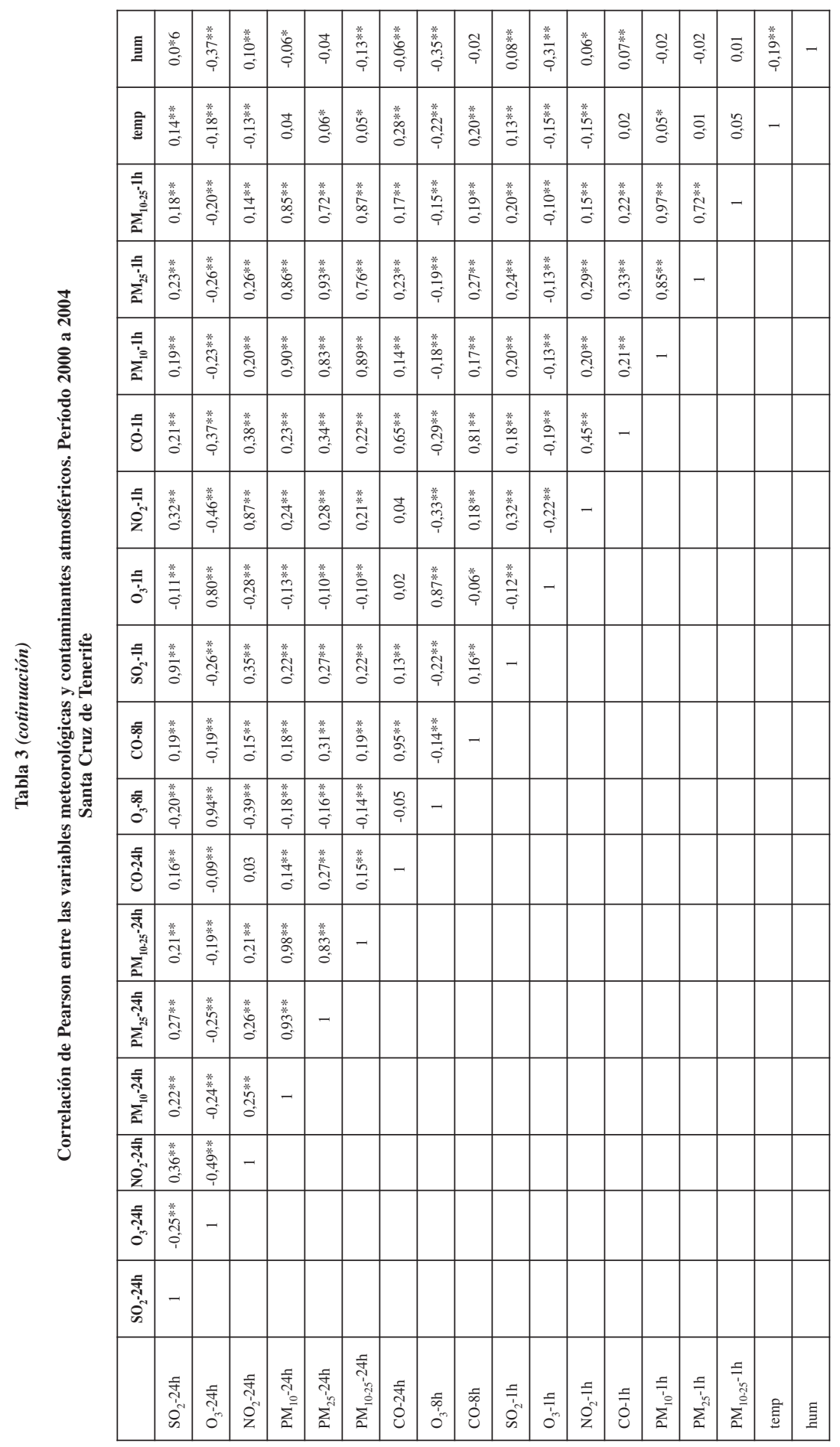


Figura 3

Distribución mensual de los valores de ozono $\left(\mu \mathrm{g} / \mathrm{m}^{3}\right)$

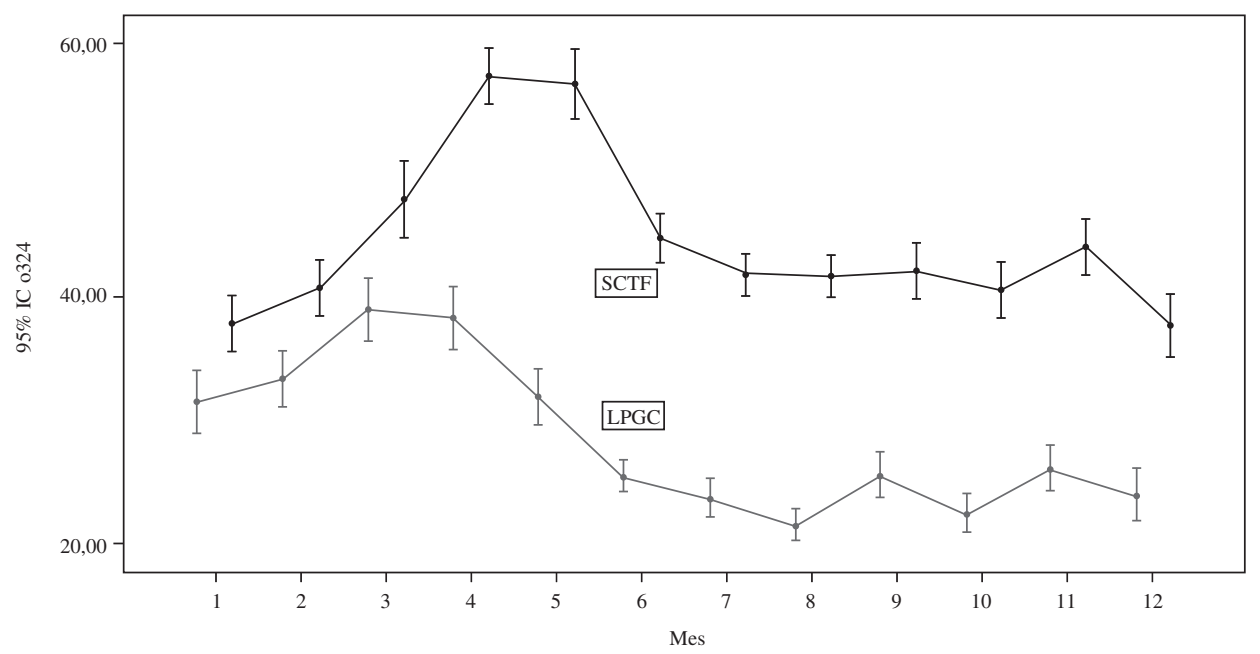

Figura 4

Distribución horaria de los niveles de $\mathrm{O}_{3}$ y $\mathrm{NO}_{2} \mu \mathrm{g} / \mathrm{m}^{3}$

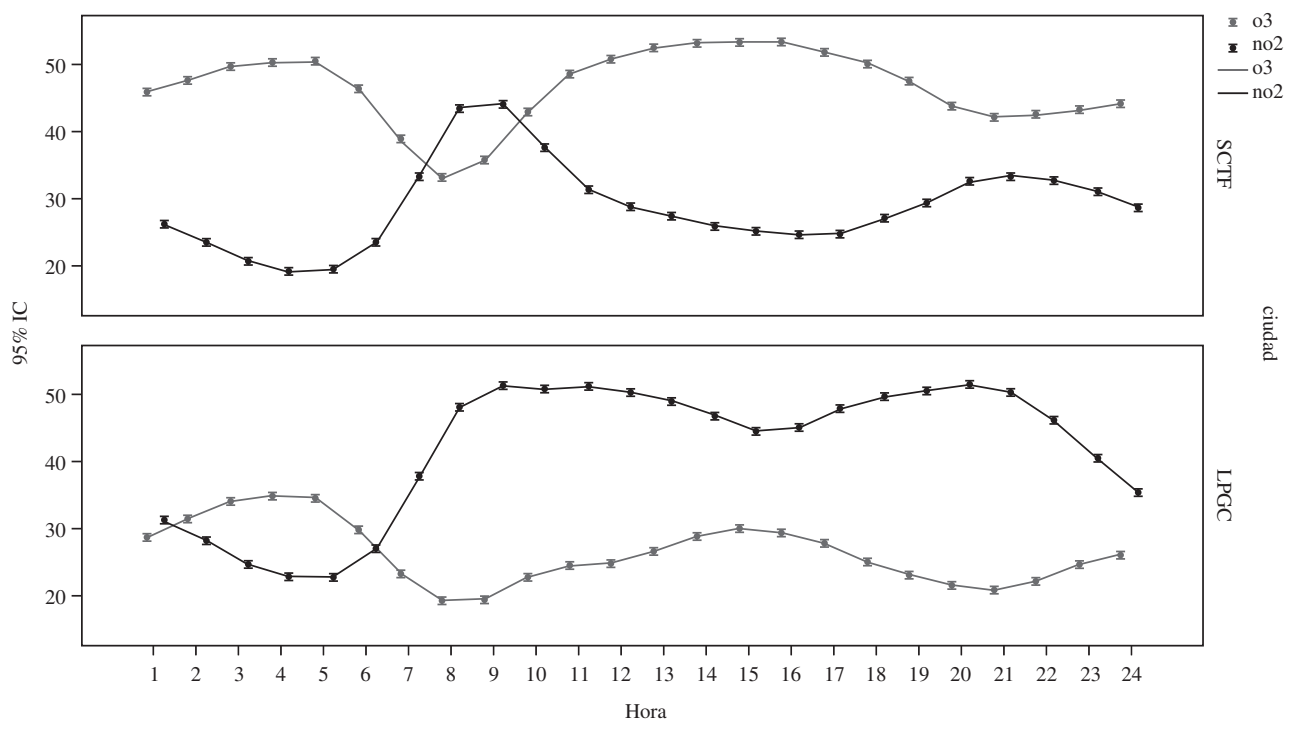

niveles de $\mathrm{SO}_{2}$ correlacionan de forma positiva aunque débil con todos los contaminantes ( $\mathrm{r}$ de Pearson: 0,37>r $>0,08$ ), excepto con los niveles de $\mathrm{O}_{3}$ con los que presenta una relación lineal inversa en ambas ciudades: $(-0,21<\mathrm{r}<-11)$; éste a su vez correlaciona de forma negativa con todos los contaminantes en Sta. Cruz de TF. Los niveles de $\mathrm{NO}_{2}$, aunque presentan una correlación positiva con el resto de 
Figura 5

Cambio porcentual de los niveles de $\mathrm{SO}_{2}, \mathrm{NO}_{2}, \mathrm{O}_{3}, \mathrm{PM}_{10}$ y $\mathrm{PM}_{2.5}$ desde 2000 a 2004
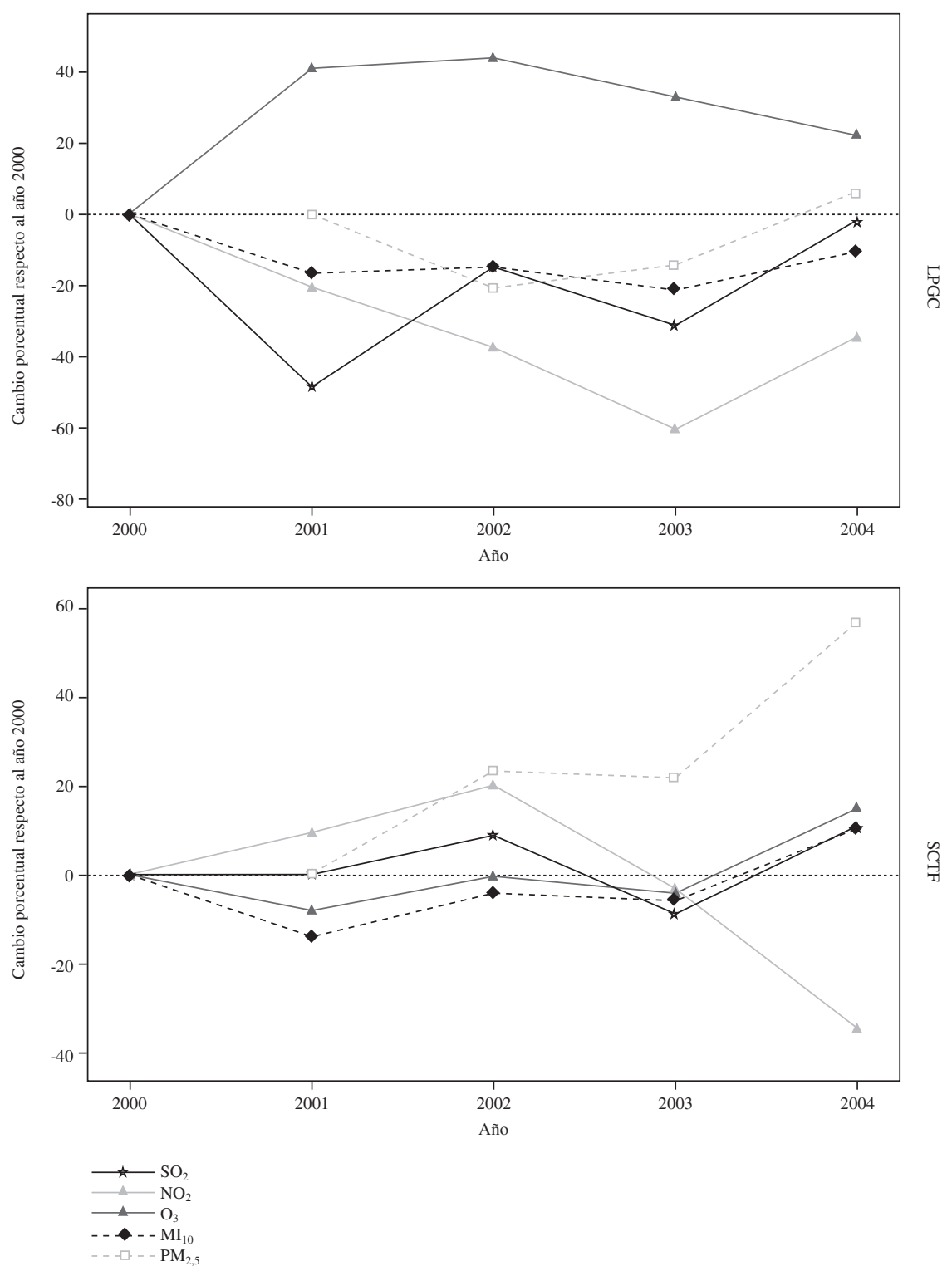

El año base es 2000 excepto para $\mathrm{PM}_{2.5}$ cuyo año base es 2001.

contaminantes, lo hace de forma negativa $-0,28<\mathrm{r}<-0,12$ con la temperatura. Existe una muy alta correlación positiva de los niveles de $\mathrm{PM}_{10}$ con las distintas fracciones de partículas ( $r>0,93$ en las dos ciudades). La correlación es algo más baja 
entre $\mathrm{PM}_{10-2,5}$ y $\mathrm{PM}_{2,5}(\mathrm{r}=0,83)$, lo que va en consonancia con lo descrito en otros estudios $^{4-8}$. Por último, los niveles de $\mathrm{PM}_{10}$ presentan una correlación débil $(r=0,22)$ con los niveles de $\mathrm{SO}_{2}$ en las dos ciudades.

\section{DISCUSIÓN}

Las intrusiones de polvo africano constituyen una fuente importante de contaminación por material particulado atmosférico en las dos ciudades canarias. Si se tiene en cuenta el conjunto de días del periodo de estudio, el aporte africano medio al promedio anual de $\mathrm{PM}_{10}$ fue de $10 \mu \mathrm{g} / \mathrm{m}^{3}$, es decir el $25 \%$ de la masa total, mientras que el aporte a los niveles promedio de $\mathrm{PM}_{2.5}$ sin influencia africana fue de 3 a $3,7 \mu \mathrm{g} / \mathrm{m}^{3}$, lo que supone un $22 \%$.

Los episodios de polvo sahariano contribuyen a la superación de los límites diarios de $\mathrm{PM}_{10}$ y $\mathrm{PM}_{2,5}$, así durante el periodo 2000 a 2004, la media diaria de $\mathrm{PM}_{10}$ ha llegado a superar los $600 \mu \mathrm{g} / \mathrm{m}^{3}$ en alguna ocasión en ambas ciudades y los $200 \mu \mathrm{g} / \mathrm{m}^{3}$ las $\mathrm{PM}_{2,5}$, habiéndose alcanzado valores máximos horarios de 1000 y $570 \mu \mathrm{g} / \mathrm{m}^{3}$ respectivamente (tabla 1). Las intrusiones tienen un patrón estacional y suceden más frecuentemente en tres momentos del año ${ }^{1,9,10}: 1$.- De enero a marzo, intrusiones de alta intensidad y de gran impacto en los niveles en superficie de $\mathrm{PM}_{10}$ y $\mathrm{PM}_{2,5}$. 2.- De junio a agosto, intrusiones en altura asociadas a ligero transporte vertical, con un aumento prolongado de niveles de fondo y de menor impacto en los niveles de inmisión de las partículas. 3.- Durante noviembre-diciembre, en superficie, de intensidad elevada y menor duración que los anteriores, con impacto en los niveles de $\mathrm{PM}_{10}$ y $\mathrm{PM}_{2.5}$. Este polvo contiene partículas minerales de calcita, dolomita, óxido férrico, cuarzo y minerales de arcilla. ${ }^{1,9}$ y afecta a todas las fracciones granulométricas de las partículas en suspensión ${ }^{9-10}$.
El límite diario de protección de la salud para los niveles de $\mathrm{PM}_{10}\left(50 \mu \mathrm{g} / \mathrm{m}^{3}\right)$ se ha superado 302 días en Sta. Cruz de TF y 211 en Las Palmas de GC. Si extraemos los días de episodio, el número de días con superación se ve reducido a 19 y 4 respectivamente. Sin embargo, aunque no se sobrepasen los 35 días que determina la normativa ${ }^{12-13}$, no se puede descartar un efecto sobre la salud en los días declarados como de intrusión de polvo africano. De un lado porque en esos días una proporción de las partículas proceden de fuentes locales (tráfico, industria), y de otra porque existen estudios que asocian efectos en salud con las partículas de origen natural ${ }^{7,8,14,15}$.

Además, en el caso del periodo estudiado para las dos ciudades, los niveles promedio anuales de $\mathrm{PM}_{2.5}$ superarían el valor guía de promedio anual para la protección de la salud establecido por la Organización Mundial de la Salud ${ }^{16}$ (10 $\mu \mathrm{g} / \mathrm{m}^{3}$ ). La singularidad en cuanto al origen y composición de las partículas a las que están expuestas las personas deben tenerse en cuenta, en primer lugar, por la manera de describir los datos de contaminación y comparar los resultados con ciudades del entorno continental, y en segundo lugar, por lo referido a las medidas de protección de la salud.

La asociación del material particulado con la salud ha sido ampliamente estudiada en las ciudades europeas, norteamericanas y asiáticas ${ }^{17-25}$. En general, los resultados de estos estudios son consistentes en el sentido de que la gran mayoría de ellos encuentran una asociación significativa en el corto plazo (es decir, entre el mismo día de la exposición y unos pocos días después) con los indicadores de mortalidad y morbilidad. Sin embargo existen menos pruebas científicas acerca del efecto que las partículas de polvo procedentes de zonas áridas o desérticas podrían tener sobre la salud $7,8,14,15,26-30$. 
Por otra parte, existen también ciertas peculiaridades en Canarias que afectan al comportamiento del ozono. Según demuestran los análisis de retrotrayectorias y los estudios realizados por diversos investigadores, los niveles de $\mathrm{O}_{3}$ se encuentran asociados a procesos de transporte a gran distancia $^{31}$, existiendo una alta correlación positiva entre este contaminante y la velocidad del viento, lo que conduce a menores niveles de NOx y consecuentemente a mayores de $\mathrm{O}_{3}$.

Lo que se ha observado durante este periodo es que los niveles más bajos de $\mathrm{O}_{3}$ se encuentran de julio a octubre y los más altos en primavera (figura 3), lo que contrasta con el patrón estacional en las capas continentales (niveles más altos en verano). Dos hechos principales determinan esta peculiaridad en el ciclo estacional del Ozono $^{1,31}$ 1.- La velocidad del viento es superior en verano por la influencia de los alisios, lo que favorece los procesos de dispersión de contaminantes locales y por tanto de los precursores del ozono. 2.- En verano con la intensificación y disminución de altura de la capa de inversión térmica, el ozono asociado al transporte a grandes distancias alcanza en menor medida la capa límite marítima, por lo que sus valores de inmisión son menores.

También existen particularidades en cuanto al ciclo diario del ozono (figura 4). Los bajos niveles de NOx durante la madrugada, que no pueden consumir el ozono transportado, y la mayor velocidad del viento de 12 a $18 \mathrm{~h}$ que favorece la dispersión de los precursores, conducen a un mayor nivel de $\mathrm{O}_{3}$ en estos dos momentos del día, aunque estos niveles no son altos comparados con otros entornos urbanos. En definitiva existe un predominio de los procesos de destrucción del $\mathrm{O}_{3}$ cuya fuente principal es el transporte oceánico y los niveles en cada ciudad, que dependerán de los aporte locales de NOx (aunque cabría esperar niveles superiores en zonas rurales o no urbanas).
Durante el periodo 2000 a 2004 no se han superado los $120 \mu \mathrm{g} / \mathrm{m}^{3} \mathrm{~N}$ de $\mathrm{O}_{3}$ (valor objetivo de protección de la salud ${ }^{13,32}$ ) en ninguna de las dos ciudades. Su concentración parece disminuir los días de episodio, bien porque aumentan los niveles de $\mathrm{NO}_{2}$ que lo consumen al empeorar la calidad del aire como se ha comentado, bien porque existe menor radiación solar debido a la elevada concentración de partículas o porque el cambio en la dirección del viento no trae $\mathrm{O}_{3}$ procedente del continente europeo.

En Sta. Cruz de Tenerife los niveles de $\mathrm{SO}_{2}$ son superiores respecto a Las Palmas de Gran Canaria, sobre todo en la estación más cercana a la zona industrial (refinería). Además cuando se examinan estos niveles los días con episodios de intrusión sahariana el promedio diario se incrementa en 10 $\mu \mathrm{g} / \mathrm{m}^{3} \mathrm{~N}$, y hasta $47 \mu \mathrm{g} / \mathrm{m}^{3} \mathrm{~N}$ el promedio de las máximas horarias diarias, mientras que en Las Palmas de GC la magnitud del incremento es menor y por lo tanto también es menor el impacto de estos eventos en la calidad del aire. Asimismo, durante el periodo 2000 a 2004, en Sta. Cruz de Tenerife el valor límite horario de protección para la salud $^{12-13}\left(350 \mu \mathrm{g} / \mathrm{m}^{3} \mathrm{~N}\right)$ se ha superado en 39 ocasiones y durante 4 días el límite diario, mientras que en Las Palmas de GC esto no ha sucedido.

En la mayoría de las ciudades los niveles de $\mathrm{NO}_{2}$ están fuertemente asociados a los de material particulado, con fuentes de emisión comunes. De hecho suele existir una alta correlación entre ellos (no es el caso de las ciudades canarias), pudiendo considerarse el $\mathrm{NO}_{2}$ como un buen indicador de la mezcla de gases y partículas emitidas por el tráfico. Además este gas es precursor de numerosos contaminantes secundarios (ácido nítrico, nitratos) y de fotoxidantes como el $\mathrm{O}_{3}$.

En Las Palmas de GC, se observa una ligera tendencia al incremento de los niveles de $\mathrm{NO}_{2}$ y el valor límite anual de $\mathrm{NO}_{2}$ $\left(40 \mu \mathrm{g} / \mathrm{m}^{3} \mathrm{~N}\right.$, vigente a partir del $\left.2010^{12-13}\right)$ 
se supera ligeramente, si bien los días en que existen intrusiones saharianas parecen tener mayor impacto en los niveles de este gas en Sta. Cruz de TF. Partiendo de la base de que en los estudios epidemiológicos es difícil distinguir el efecto independiente de este gas sobre la salud, aportarían más información aquéllos que se realizan en ciudades con distribución temporo-espacial de $\mathrm{NO}_{2}$ y material particulado diferente, y en aquellas ciudades en las que los niveles de $\mathrm{NO}_{2}$ no están correlacionados con partículas, condición que parece cumplirse en ambas ciudades.

La información anterior da una idea de los patrones de exposición a los contaminantes atmosféricos en ambas capitales canarias durante el periodo 2000 a 2004. Por otra parte, existe una marcada estacionalidad en la exposición a las partículas ambientales y al ozono, con ciclos y características que las diferencian de ciudades de otros entornos. El impacto natural de las partículas procedentes del continente africano es de tal magnitud en la composición y niveles anuales de partículas que no existen diferencias notables entre las dos ciudades, tan sólo un mayor predominio, de la fracción $\mathrm{PM}_{10}$ en Sta. Cruz de Tenerife, donde parece que la calidad del aire se ve más afectada ante este fenómeno probablemente por la orografía que la rodea y por la sinergia con las fuentes de emisión industrial. Sin embargo, la exposición al resto de contaminantes tiene características propias en cada una de las dos ciudades. Así los niveles medios de $\mathrm{SO}_{2}$ son superiores en Sta. Cruz de Tenerife lo que orienta a una mayor influencia industrial, mientras que en Las Palmas de Gran Canarias, con un mayor impacto del tráfico rodado, los valores promedio de $\mathrm{NO}_{2}$ son más elevados.

De igual modo, hay que tener en cuenta que, debido a la climatología del archipiélago, especialmente en estas ciudades ubicadas junto al mar, la ventilación en los hogares es natural, apenas existe uso de sistemas artificiales de climatización y la gente pasa muchas horas al día en el exterior. Por ello las mediciones realizadas en la red dan una idea bastante cercana al promedio de la exposición de sus ciudadanos, sobre todo en lo referente a las partículas y al $\mathrm{O}_{3}$ que en parte son contaminantes secundarios que se distribuyen de forma más uniforme que el resto y en el caso de estas ciudades además habría que tener en cuenta el gran impacto $\mathrm{y}$ uniformidad de las $\mathrm{PM}_{10}, \mathrm{PM}_{10-2,5} \mathrm{y}$ $\mathrm{PM}_{2,5}$ de origen natural.

En conclusión, en las dos ciudades canarias más pobladas se ha identificado un patrón de contaminación atmosférica caracterizado por una gran influencia de su localización geográfica (cercanía a las mayores zonas desérticas del continente africano), orografía y meteorología. Todo ello determina un perfil de calidad del aire marcado por episodios con niveles muy altos de partículas de origen natural, así como una estacionalidad diferente a la de otras ciudades respecto al ozono, junto a un patrón de contaminación urbano-industrial en Sta. Cruz de Tenerife y netamente urbano en Las Palmas de Gran Canarias.

Desde el punto de vista de la salud pública es necesario tener en cuenta estos resultados a la hora de establecer sistemas adecuados de vigilancia, así como para analizar su posible impacto en la salud de las personas de las Islas Canarias.

\section{BIBILIOGRAFÍA}

1. Viana M, Querol X, Alastuey A, Cuevas E, Rodríguez S. Influence of African dust on the levels of atmospheric particulates in the Canary Island air quality network. Atmos Environ. 2002; 36 (38): 5861-75.

2. Ministerio de Medio Ambiente, Instituto de Ciencias de la Tierra, CSIC, CIEMAT, CEAM, Instituto de Salud Carlos III. Estudio y evaluación de la contaminación atmosférica por material particula- 
do en España: Desarrollo metodológico para estudio de las series temporales de partículas. Madrid: Ministerio de Medio Ambiente; 2001.

3. EC Working Group on Particulate matter. Guiadance to member states on PM10 monitoring and intercomparisions with the reference method. Final report: Methodology for the evaluation of the impact of natural events on PM10 levels. Brussels: UE; 2002

4. Burnett, R. T et al Association between particulate and gas-phase components of urban air pollution and daily mortality in eight Canadian cities. Inhal Toxicol. 2000; 12:15-39.

5. Lippmann M, Frampton M, Schwartz J, Dockery $D$ et al. The U.S. Environmental Protection agency particulate matter health effect research Centers program: a midcourse report of status, progress and plans. Environ Health Perspect. 2003; 111: 1074-10.

6. Lippmann, M., Ito K, Nadas A, Burnett RT. Association of particulate matter components with daily mortality and morbidity in urban populations. Boston, MA. Res Rep Health Eff Inst. 2000; 95: 5-72.

7. Mar T, Norris GA, Koenig JQ, Larson T. Association between Air Pollution and mortality in Phoenix, 1995-1997. Environ Health Perspect. 2000; 108 (4): 347-352.

8. Cifuentes LA, Vega J, Köpfer K, Lave LB. Effect of the Fine Fraction of Particulate Matter versus the Coarse Mass and Other Pollutants on Daily Mortality in Santiago, Chile. J Air \& Waste Manag Asso. 2000; 50: 1287-1298.

9. Viana MM. Niveles, composición y origen del material particulado atmosférico en los sectores norte y este de la Península Ibérica y Canarias [Tesis Doctoral]. Barcelona: CSIC; 2003.

10. Querol X, Alastuey A, Viana MM, Rodriguez S, Artiñano A, Salvador P, García do Santos, Fernández Patier R, Ruiz CR, de la Rosa J, Sánchez de la Campa, Menéndez M, Gil JI. Specification and origin of pm10 and pm2.5 in Spain. J Aerosol Sci. 2004; 35: 1151-1172.

11. Claiborn C S,Finn D,Larson T V,Koening J Q. Windblown Dust Contributes to High PM2.5 Concentrations. J Air Waste Manage Assoc. 2000; 50:1440-1445.

12. Boletín Oficial del Estado. Real Decreto 1073/2002, de 18 de octubre, sobre evaluación y gestión de la calidad del aire ambiente, en relación con el dióxido de azufre, dióxido de nitrógeno, óxidos de nitrógeno, partículas, plomo, benceno y monóxido de carbono. BOE núm. 260 de 30/10/2002.

13. Diario Oficial de las Comunidades Europeas. Directiva 2008/50/CE del Parlamento Europeo y del Consejo, de 21 de mayo de 2008 relativa a la calidad del aire ambiente y a una atmósfera más limpia en Europa. DO L152/1 de 11/06/2008.

14. Castillejos M, Borja-Avurto V, Dockery D, Loomis D. Coarse particles and mortality in Mexico City. Inhal Toxicol. 2000; 12 (1): 61-72.

15. Burnett R. T, Brook J, Dann T, Delocla C, Philips $\mathrm{O}$, Cakmak $\mathrm{S}$ et al. Association between particulate and gas-phase components of urban air pollution and daily mortality in eight Canadian cities. Inhal toxicol. 2000; 12:15-39.

16. Organización Mundial de la Salud. Guías de la Calidad del Aire relativas al material particulado, el ozono, dióxido de nitrógeno y dióxido de azufre. Actualización mundial 2005. Resumen de evaluación de los riesgos. Ginebra: OMS; 2006.

17. Katsouyanni et al Short-term effects of ambient sulphur dioxide and particulate matter on mortality in 12 European cities: results from times series data from the APHEA project. BMJ. 1997; 314: 1658-1663.

18. Ballester F, Iñíguez C, Saez M, Pérez-Hoyos S, Daponte A, Ordóñez JM, et al. Relación a corto plazo de la contaminación atmosférica y la mortalidad en trece ciudades españolas. Med Clin (Barc) 2003; 121:684-9.

19. Ballester F, Rodríguez MP, Ïñiguez C, Sáez M, Daponte A, Ordóñez JM.. The EMECAM/EMECAS Project a Spanish Multicentre Study on Health Effects of air Pollution. WHO: Newsletter. 2001; 33: 8-14.

20. Biggeri A, Bellini P, Terracini B, (editors). Metaanalysis of the Italian Studies on Short-term Effects of Air Pollution. 2001; Epidemiol Preven 25(2).

21. Samoli E et al Sensitivity analyses of regional differences in short-term effects of air pollution on daily mortality in APHEA cities. In: Revised analyses of time time-series studies of air pollution and health. Special report. Boston, MA: Health Effects Institute; 2003

22. Ballester, F., M. Saez, S. Perez-Hoyos, C Iñíguez, A Gandarillas, A. Tobias, J. Bellido, M Taracido, F. Arribas, A. Daponte, E. Alonso, A Cañada, Guillen- 
Grima F., Ll. Cirera, M. J. Perez-Boíllos, C Saurina, F Gómez, J. M. Tenías, and on behalf of the EMECAM group. The EMECAM project: a multicenter study on air pollution and mortality in Spain. Combined results for particulates and for sulphur dioxide. Occup Environ Med 2002;. 59: 300-308.

23. Stieb DM, Judek S, Burnett RT Meta-Analysis of Time-Series Studies of Air Pollution and Mortality: Effects of Gases and Particles and the Influence of Cause of Death, Age, and Season J. Air Waste Manage. Assoc. 2002; 52:470-484.

24. Samet JM, Zeger SL, Dominici F, Curriero F, Coursac I, Dockery DW, Schwartz J, Zanobetti A. The National Morbidity, Mortality, and Air Pollution Study. Part II: Morbidity and Mortality from Air Pollution in the Unites States. Boston, MA, Health Effects Institute/Research Report 94, Part II; 2000.

25. Venners SA, Wang B, Peng Z, Xu Y, Wang L, Xu X. Particulate Matter, Sulfur Dioxide, and Daily Mortality in Chongqing, China. Environ Health Perspect 2003; $111: 4$.

26. Schwartz J, Norris G, Larson T, Sheppard L, Clairborne C, Koenig J. Episodes of high coarse particle concentrantions are not associated with increased mortality Environ Health Perspect. 1999; 107 (5):339-342
27. Gyan K, Henry W, Lacaille S, Laloo A, LamseeEbanks C, McKay S, Antoine RM, Monteil MA African dust clouds are associated with increased paediatric asthma accident and emergency admissions on the Caribbean island of Trinidad. Int $\mathbf{J}$ Biometeorol. 2005; 49: 371-376

28. Brunekreef, Forsberg B Epidemiological evidence of effects of coarse airborne particles on health. Eur Respir J 2005; 26: 309-318.

29. Yang CY, Chen YS, Chiu HF, Goggins WB. Effects of Asian dust storm events on daily stroke admissions in Taipei, Taiwan Environ Research. 2005; 99: 78-84.

30. Mar T, Norris GA, Koenig JQ, Larson T. Association between Air Pollution and mortality in Phoenix, 1995-1997. Environ Health Perspect. 2000; 108 (4): 347-352.

31. Rodríguez S, Guerra JC. Monitoring of ozone in a marine environment in Tenerife (Canary Islands). Atmospheric Environ. 2001; 135: 18291841.

32. Boletín Oficial del Estado. Real Decreto $1796 / 2003$, de 26 de diciembre, relativo al ozono en el aire ambiente. BOE núm. 11 de 13/01 2004. 